and bone marrow transplantation and a final chapter discusses immunosuppressive therapy in relation to general medical diseases.

Immunosuppressive therapy is a field which is only 20 years old and important and major changes occur with great rapidity; it is therefore difficult even with a book of this kind to have kept astride of new events with delays in publication. However, the book has collated the historical aspects, the development of the field and the present ideas on this advancing subject in a comprehensive way.

The book is useful for clinicians involved in transplantation, since it integrates the experience of different centres of grafting and describes techniques and difficulties in the individual disciplines. Inevitably, there will be some overlap between chapters on drug usage and side effects but overall each chapter has been written by an author expert in his field and in general each is concise and informative with a balance between established factual data and ongoing experimental work.

The book is well presented and introductions and conclusions at the beginning and end of each chapter make it very readable. The bibliography following each chapter is extensive and the index sound. The book is recommended for both general reading and reference, and achieves its aim as an overall review of immunosuppressive therapy in clinical situations.

\section{Rapid Virus Diagnosis. Application of Immunofluorescence}

By P. S. Gardner and J. McQuillin. 2nd edn, Pp. xiii +317 , illustrated. Butterworths, London, 1980. $£ 29.00$ Rapid diagnosis of virus infections is of considerable importance for several reasons: it is essential if specific antiviral drugs are to be administered at an early stage of the illness in the differential diagnosis of syndromes such as aseptic meningitis or respiratory disease early demonstration of a viral aetiology may remove the need to administer unnecessary, and often toxic, drugs; when viruses present hazards to the community, rapid diagnosis is of importance in indentifying and localizing infected cases. Immunofluorescence has become an important method for rapid diagnosis of a number of virus infections and, provided that the techniques are carefully controlled, the results are reliable and may be reported to the clinician a few hours after sampling. Mistakes occur if 'short cuts' are taken and care must be exercised in the preparation and standardization of reagents and in the incorporation of adequate controls in the test.

This book provides an excellent laboratory handbook and reference source for virologists who are interested in the uses of immunofluorescent staining for research work and more particularly for diagnosis. The first chapter, on the fluorescence microscope and basic principles, is followed by 3 chapters on reagents, techniques and preparation of specimens. The remainder of the book is concerned with detailed descriptions of the uses of the techniques in all aspects of virus infections for which they are a practical proposition. In the introduction it is stated that the book is intended 'not only for the physician and medical student but also for the laboratory technician and medical scientist'. I suggest that it will be welcomed by clinical virologists, medical laboratory scientific officers and some research workers. It would be very optimistic to expect that physicians and medical students will buy such a specialized volume which covers only one aspect of rapid virus diagnosis. It is difficult enough to persuade medical students to invest in a suitable textbook of medical virology and perhaps this is inevitable with the current prices of books.
Recent Advances in Clinical Psychiatry. No. 3

Edited by K. Granville-Grossman. Pp. ix +339 , illustrated. Churchill, Livingstone, Edinburgh, London and New York, 1979. £15.00.

Research in psychiatry continues to be an expanding field. With time and energy it may be possible to keep in touch with progress through journals alone. For most, however, it is an advantage to have access to a source that brings recent publications together in a logical and informed manner. Dr Granville-Grossman's series does this admirably and number 3 is well up to standard.

Some 15 authors, 'acknowledged experts' in their own fields are brought together to present 10 chapters, ranging from Transcultural psychiatry to Anxiety states. The first chapter on research on schizophrenia is typical of the standard throughout. Each presentation is really an up-to-date review, rather than merely a presentation of recent papers and, as a result, should be of value to a wide variety of clinicians not only in the field of psychiatry. The book does have a very strong organic bias, but this probably reflects the pace of progress with modern biochemical and epidemiological techniques. Occasionally, this leaning does detract a little as, for example, the 2 references to extrapyramidal side effects of psychotropic drugs are somewhat divorced from clinical relevance.

Generally the criticisms are minor and this book is to be widely recommended and perhaps mandatory reading for psychiatrists and physicians in training.

\section{Rheumatic Disorders in Childhood}

By Barbara M. Ansell. Postgraduate Paediatrics Series -General Editor: J. Apley. Pp. vii +299 , illustrated. Butterworths, London, 1980. $£ 25.00$.

Recurrent limb pains, cramp, and backache are all common symptoms in children. They may be easily dismissed, yet occasionally they can herald the onset of a chronic problem. This book provides a practical approach to the recognition and assessment of specific joint disorders in childhood. It draws on a wealth of experience in the field, placing emphasis on accurate assessment of the history and clinical findings, and discussing the approaches to investigation and management which will be of greatest benefit to the child. It is profusely illustrated with both radiographs and photographs, including a colour section; it is well indexed and referenced.

The opening chapter examines the spectrum of vague pains of non-rheumatological origin, there follow chapters on monarticular arthritis, polyarthritis, and juvenile chronic arthritis. Rheumatic fever retains a section of its own, and connective tissue disorders, haemoglobinopathies, and various oddities are also treated separately. The terminology used in the classification of childhood arthritis has long been regarded as obscure to the non-specialist, nevertheless, careful study of this book clarifies the nomenclature in a positive way. The use of the term rheumatoid is deplored, the concept of juvenile chronic arthritis is emphasized and distinguished from the many other causes of an arthropathy. The importance for prognosis within juvenile chronic arthritis of the patterns of joint and systemic presentation, sero-positivity and HLA status is thoroughly discussed. An appendix outlining laboratory tests is inserted. The principles of management at different ages, and the impact on family, school, and social life are covered sensitively. The place of rest, splinting, physiotherapy, and drugs is reviewed. The uses of analgesics, anti-inflammatory agents, slow acting drugs such as gold and penicillamine, and immunosuppressive therapy are described in relation to their use at Taplow. It is of great interest that the experience of this unit has now been crystallized in this way; it will be of great value to 